\title{
Intentional Unilateral Sacral Epidural
}

\author{
Radhashyam Paria ${ }^{1}$, Smarajit Surroy ${ }^{1}$, Mousumi Majumder ${ }^{1}$, Baishakhi Paria ${ }^{2}$, \\ Soma Sengupta ${ }^{3}$, GoutamDas, ${ }^{4}$ Anshuman Paria ${ }^{5}$ \\ 1(Dept of Anesthesiology, Howrah Orthopedic Hospital, Eastern Railways, WB) \\ 2(Department of Community Medicine, National Medical College) \\ 3(dept of Anesthesiology, SSKM hospital, WB) \\ 4(Department of Pediatric Medicine, North Bengal Medical College, West Bengal) \\ 5(Dept of Neonatology, SSKM hospital, WB)
}

\begin{abstract}
Background: Unilateral block by lumbar epidural and caudal anesthesia is a rare event, although, from time to time, it becomes essential for aged patients to minimize the drug effect and to have full benefits of epidural anesthesia.

Aims: This study is scheduled to get a way to achieve unilateral lower limb block with the help of the sacral epidural anesthesia.

Method: $25 \mathrm{ml}$ of 0.5\% Ropivacaine were injected into sacral epidural space in the lateral position with slight head down tilt. The patient was kept in the same position on the operation table for 15 minutes after completion of sacral epidural anesthesia on the surgical side with the nerve stimulator needle used for detection of the epidural space.

Result: All patients expect five developed unilateral lower limb block, placed on the operation table and projected for surgery. Other five patients developed bilateral block.

Conclusion: The sacral epidural through its dorsal foramen in lateral position of the patient is highly capable of providing the unilateral block on dependent lower limb for projected surgeries without hemodynamic fluctuation.
\end{abstract}

Key words: dorsal foramen, nerve stimulator needle, lumbosacral segment, sacral epidural, lumbar epidural.

\section{Introduction:}

Development of unilateral block of lower limb during the procedure of lumbar epidural or caudal anesthesia is a rare occurrence. Intentionally, such incidence is impossible to occur with a conventional technique of epidural anesthesia. Sometimes it is found as complication of lumbar epidural due to slow injection of small volume of local anesthetic, presence of congenital median epidural septum; $(1,2)$ acquired midline adhesion; $(3,4)$ misplacement of the catheter tip into the para vertebral area; and also in the anterior epidural space (5-10). In some occasions, unilateral block is found to develop in caudal anesthesia, most probably due to misdirected needle (11) or the presence of congenital fibrous/ connective tissue band.(12) All the above causes are responsible for the development of unilateral block as complication of epidural technique. On the other hand, unilateral block during lower limb surgery contributes unlimited beneficial effects in connection of hemodynamic stability. It provides the facility of minimal systemic disturbance and less involvement of spinal segments, responsible for hemodynamic regulation of the body. Such beneficial facility is not usually available during the conventional procedure of intrathecal and epidural technique of anesthesia. So we hypothesized to administer sacral epidural anesthesia through its dorsal foramens on lateral position of the patient placing surgical side on the operation table to evaluate advantages and disadvantages of this technique.

\section{Methods:}

With the approval of Medical Ethical Review Board and written informed consent for procedure and study, 30(Thirty) patients belonging to ASA class 1 and 11 were selected for surgeries of lower limb under unilateral sacral epidural anesthesia.

Their consents were signed after full explanation about the expected benefits and results, side effects and complications of the technique of sacral epidural through its dorsal foramens. Every participant was subjected to preoperative visit to exclude the contraindication of the proposed procedure.

In the O.T, peripheral infusion and non -invasive monitoring were started. Anatomical landmarks were identified. We identified the spinous process of fourth lumbar vertebra (L4) and tip of coccyx. Next, we located the mid-point of above two landmarks as third sacral vertebra (S3). The first sacral vertebra (S1) was located as mid-point of L4 and S3. The second sacral vertebra (S2) was found at the mid-point between S1 and S3. The spinous processes of $\mathrm{S} 1$ and $\mathrm{S} 2$ are found approximately $2.5 \mathrm{~cm}$ apart, and their respective foramina lie $1.5 \mathrm{~cm}$ caudal and lateral to them. Similarly, S3 foramina lie $1.5 \mathrm{~cm}$ caudal and lateral to S3 vertebra. 
After identification of S1, S2 and S3 foramina, aseptic preparation was done in the lateral position of patients keeping surgical side downward on the operation table, and selected foramens were infiltrated with $6 \mathrm{ml}$ of $1 \%$ injection lignocaine. We inserted the nerve stimulator need [13] (0.8 mm x $100 \mathrm{~mm} 21 \mathrm{G}$ Stimuplex A100 needle/ BBraun) through any of the three dorsal foramens selected beforehand. The needle was advanced perpendicularly like sacral spinal anesthesia [14,15] until it contracted with bone. The depth of needle was noted from grazed nerve stimulator needle and needle was withdrawn a little and again redirected 40 degrees to the foramen until it entered the foramen and crossed the intervertebral foramen evidenced with movement of great toe on the same side. Thus, we placed the needle correctly in the sacral epidural space. This was also further confirmed by loss of resistance and absence of cerebrospinal fluid (CSF) and blood in the needle. After confirmation of needle placement in sacral epidural space, $25 \mathrm{ml}$ of $0.5 \%$ Ropivacaine was injected through the needle (2.5ml / segment of the vertebra) [17] and keeping patient on the same position for another ten minutes and after which patient was turned to the supine position. After eliciting sensory block height suitable for proposed surgery, surgeons were allowed to operate. Another leg was examined to detect the involvement of block.

Heart rate, blood pressure, respiration, and oxygen concentration were recorded every three minutes for first ten minutes after which taken at every ten minutes interval. In the case of lower limb surgery upper level of sensory block was assessed by pinprick and motor block by modified Bromage scale which is as follows: $0=$ lifting up extended leg, one = flexed knee with full ankle movement, 2 No knee movement, partial ankle movement, 3 = complete paralysis. We also recorded the onset time of sensory and motor block as the time gap between the epidural injection and bilateral loss of sensation and loss of motor activity of the lower limbs respectively. Similarly, we calculated the duration of the sensory and motor block and sent all collected data for statistical analysis using Graphpad Prism 5.

\section{Results:}

30 elderly patients were administered unilateral lower limb block intentionally in the lateral position with slightly head down tilt of the O.T. table. Out of 30 patients, 5 patients developed bilateral block (16.66\%). Rest of patients developed unilateral block on surgical side. All patients possessed stable hemodynamic status and profound analgesia throughout intraoperative period. The delayed onset of motor and sensory block was same as epidural anesthesia. The intentional unilateral block technique with help of sacral epidural anesthesia was not associated with incomplete block. The nerve block profile is cited in table no 1. All patients were not accompanied with cardiovascular fluctuation and hypoxia. Their hemodynamic and demographic profile are described on the table no 1.

\section{Discussion:}

The development of intentional unilateral lower limb block by sacral epidural through its dorsal foramen is a simple procedure of single shot administration of local anesthetic in sacral epidural space in the lateral position of the patient and keeping the patient on the same position for another ten minutes. It provides less physiological disturbance like brachial plexus block in upper extremity with avoidance of cardiovascular instability. This technique of anesthesia is highly appropriate for the elderly patient of lower limb's surgery.

The intentional development of unilateral lower limb block by sacral epidural is highly effective and beneficial although it is a time-consuming procedure with requirement of large volume of local anesthetic like other peripheral nerve block (femoral, sciatic and brachial plexus block). The supplementary analgesic may be essential in case of incomplete block.

The motor and sensory activities of lower limbs depend on lumbosacral roots of the spinal cord extending from the first lumbar spinal segment to fifth sacral spinal segments (L1 to S5). Similarly, the sympathetic supply to the lower limbs depends on neurones in the lateral horn of the lower three thoracic (T10, T11 and T12) and upper two lumbar (L1 L2) spinal segments and are responsible to control the hemodynamic status of lower limbs. The unilateral segmental block of the lumbosacral roots of spinal cord achieved by unilateral sacral epidural provides more hemodynamic stability to patients.

Problematic situation arises in anesthetic management of elderly patients owing to their limited organ reserve and coexisting compromised cardio-respiratory status. Neuraxial block has definitive benefits in better control of surgical stress as well as a reduction in blood loss during surgery.

The anatomical changes of the sacral hiatus due to bony overgrowth along with soft tissue obscure the usual landmarks of the sacral hiatus and thus entry of the needle through the hiatus becomes impossible in adult life. The absence of the sacral hiatus in $7.7 \%$ of population further contributes the failure of caudal anesthesia. Similarly, the absence of the sacral canal in 5\%-10\% population further leads to the failure of classical caudal. The results of such repeated failure of caudal in adults through sacral hiatus led to near abolition of this technique. The sacral epidural through its foramen completely removed the difficulties faced during adult caudal anesthesia. 
Bilateral first three pairs of dorsal foramens are selected to locate the epidural space within sacral canal, but sometimes it becomes difficult due to anomalies of foramina. These foramina may be smaller or even obliterated due to deposition of calcium around the rim. Usually, 3rd dorsal foramen of sacrum is mostpreferable site to insert nerve stimulator needle owing to less chance of dural puncture. The 1 st and $2 \mathrm{nd}$ dorsal foramens of sacrum are also a place of choice for easy introduction of nerve stimulator needle with potential danger of dural puncture. The first three pairs of dorsal foramens are suitable to introduce needle to sacral epidural space although, it is not sure to get it at the first attempt, may need more or fail to get. In that event, it is better to leave it and attempt for another one. The immobility of sacrum helps to introduce the nerve stimulator needle in the proper direction without any help of an assistant, and it also helps in quick healing of the puncture site without causation of backache.

The large capacity of sacral epidural space can accommodate the large volume of local anesthetic. In addition, free leakage of local anesthetic through its multiple connections needs more solution. It is similar as caudal and roughly advocated as $2-3 \mathrm{ml} /$ segment to block. [17]

The cranial spread of lumbar epidural anesthesia is somewhat more extensive than caudal spread. Indeed, the block of the fifth lumbar (L5) and first sacral (S1) segments by local anesthetics is often delayed or incomplete. It has been reported that the delay in onset at (L5) and (S1) segments may be because of the large size of nerve roots. However, there is a large degree of inter-individual variability in root size. But in case of sacral epidural, lumbosacral segments of spinal cord have been blocked completely with limited cranial spread and maximal cardiovascular stability.

Sacral epidural space (SES) is continuous upwards as lumbar epidural space and below as sacral hiatus. Thus after injection of local anesthetic in SES, reduced height of the cephalad flow of local anesthetic is more likely due to free leakage through patent openings and large capacity of SES. The capacious sacral epidural space helps to explain the less involvement of spinal segments

Lumbosacral spinal nerve roots (LSNR), being a vital component for the innervations of lower limbs is specifically blocked by sacral epidural with restricted cephalad extension and providing profound analgesia. The technique of sacral epidural through foramen is highly efficient to have segmental block of LSNR for lower limbs surgery avoiding difficulties faced during administration of caudal in elderly patients. This procedure is also capable of providing high-quality analgesia in the period immediate after operation to counteract the surgical stress response.

The sacral epidural through its dorsal foramens is similar to the effects of conventional caudal introduced through sacral hiatus. Only routes of approach are different. One is through dorsal foramen, and other is through hiatus. Both have same site of action on the same neural components. In fact, the sacral epidural anesthesia may be regarded as 'modified caudal'.

The use of nerve stimulator needle, [13] in place of the epidural needle, helps to detect the epidural space correctly with evidence of great toe movement. It, being smaller in diameter comparing with conventional type of epidural needle, causes less nerve injury at the time of introduction through sacral intervertebral foramen. It is also beneficial to limit the extent of the nerve stimulator needle within the epidural space with avoidance of dural puncture.

The local anesthetic, deposited in the lumbar epidural space is associated with different types of spread of local anesthetic. The cephalic and caudal movement of local anesthetic is associated with longitudinal spread along the epidural space. The circumferential spread of local anesthetic is the key factor for the development of bilateral sensory block.[18] The cephalic and caudal spread of local anesthetic is responsible for extension of block. But during the procedure of sacral epidural anesthesia in the lateral position of the patient keeping surgical side on the operation table is associated only with longitudinal spread of local anesthetic without circumferential spread of local anesthetic. Actually, the lower half of the sacral canal in longitudinal direction is fulfilled with local anesthetic and nerve roots of the same half are immersed with local anesthetic. The free leakage of local anesthetic through the dorsal foramens of the same side avoids the involvement of nerve roots of upper side. Thus, the nerve roots of the upper half of the sacral canal remains spared from the solution.

All participants of this study are aged with some degree of cardio-respiratory compromise with or without hepatic and renal insufficiency. Their residual reserve organs lead to many anesthetic problems. Cardiovascular fluctuation owing to conventional methods of neuraxial block is always associated with physiological disturbance of the body. The unilateral lower limb block, developed intentionally by sacral epidural anesthesia through its foramen provides hemodynamic stability.

\section{Conclusion:}

The sacral epidural anesthesia through its dorsal foramen is efficiently capable of producing unilateral lower limb block on dependent side in lateral position of patient without cardiovascular fluctuation in $83.3 \%$ cases. 


\section{References:}

[1]. Gallart L, Blanco D, Samso E, Vidal F. Clinical and radiologic evidence of the epidural plica mediana dorsalis. Anesth Analg 1990;71:698-701.

[2]. Peduto VA, Tani R, Marinelli L, Pani S. Bilateral analgesia and unilateral paresis after lumbar epidural blockade. Anesth Analg 1992;74:294-6.

[3]. Singh A. Unilateral epidural analgesia. Anaesthesia 1967;22:147-9.

[4]. Yamagami H, Yuda Y, Shiotani M, et al. The administration of continuous epidural block under prone position with fluoroscopic guidance. Masui 1989;38:229-35.

[5]. Usubiaga JE, Reis A, Usubiaga LE. Epidural misplacement of catheters and mechanisms of unilateral blockade. Anesthesiology 1970;32:158-61.

[6]. Hehre FW, Sayig JM, Lowman RM. Etiologic aspects of failure of continuous lumbar peridural anesthesia. Anesth Analg 1960;39:511-7.

[7]. Kawaguchi T, Inoue S, Fukunaga A. Clinical analysis of anesthetic failure of continuous lumbar epidural anesthesia. Masui 1966;15:1130-6.

[8]. Shanks CA. Four cases of unilateral epidural analgesia. Br J Anaesth 1968;40:999-1002.

[9]. Andoh K, Shima T, Hoshi K, Hashimoto Y. An X-ray study of the catheters abnormally placed in the epidural space. Masui 1991;40:1598-1602.

[10]. Asato F, Hirakawa N, Oda M, et al. A median epidural septum is not a common cause of unilateral epidural blockade. Anesth Analg 1990;71:427-9.

[11]. Bernadette VT, Michael CJ. Epidural Neural Blockade. In: Michael CJ, Phillip BO, editors. Cousins and Bridenbaugh's Neural Blockade in Clinical Anesthesia and Pain Medicine. 4 th ed. Philadelphia: Lippincott, Williams \& Wilkins; 2009. p. 285. Back to cited text no. 1

[12]. Withington DE, Weeks SK. Repeat epidural analgesia and unilateral block. Can J Anaesth 1994;41:568-71. Back to cited text no. 2 [PUBMED]

[13]. Tsui, B.C., Tarkkila, P., Gupta, S., Kearney, R. Confirmation of caudal needle placement using nerve stimulation. Anesthesiology 91: 374; 1999.

[14]. Paria R, Surroy S, Majumder M, Paria A, Paria B, Das G. Sacral spinal anesthesia. Indian J Anesth. 2014; 58(1) : 80-2

[15]. Paria R, Surroy S, Majumder M, Paria B, Sengupta S, Paria A. Sacral Saddle Block IOSR-JDMS 2014; 13(4) 39-40.

[16]. Paria R, Surroy S, Majumder M, Paria B, Sengupta S, Paria A. Sacral Epidural Anesthesia IOSR-JDMS 2014; 13(5) 10-11.

[17]. Capt STEVEN J.ZITO, CRNA, BS, USAF, NC. Adult caudal anesthesia: A reexamination of the technique. Journal of the American Association of Nurse Anesthetists. 1993; 61(2): $153-7$.

[18]. Usubiaga JE, Reis A, Usabiaga LE. Epidural misplacement of catheters and mechanisms of unilateral blockade. Anesthesiology 1970; 32:158-61.

\begin{tabular}{|lc}
\hline Table no 1 Showing Demographic profile, Hemodynamic profile and Nerve block profile of 30 patients. \\
DEMOGRAPHIC PROFILE & $77.27 \pm 5.39$ \\
Age (years) & $16: 14$ \\
Height (Cm) & $59.70 \pm 3.85$ \\
Weight (Kg) & $158.53 \pm 4.48$ \\
Sex (M:F) & $113.87 \pm 6.2$ \\
& $78.37 \pm 4.63$ \\
HEMODYNAMIC PROFILE. & $98.93 \pm 0.9$ \\
Heart Rate (beats/min) & \\
Oxygen Saturation (\%) & 83.3 \\
NERVE BLOCK PROFILE: & $\mathrm{T}_{12}$ \\
Success rate (\%) & $25.10 \pm 3.35$ \\
Onset time of sensory block.(min) & $329.13 \pm 7.41$ \\
Onset time of motor block.(min) & $284.20 \pm 11.62$ \\
Duration of sensory block. (min) & $22 . \pm 1.32$ \\
Duration of motor block.(min) & \\
Cephalad extension. (vertebra & \\
\hline
\end{tabular}

